Bull. Korean Math. Soc. 48 (2011), No. 3, pp. 637-646

DOI 10.4134/BKMS.2011.48.3.637

\title{
COMPETITION INDICES OF STRONGLY CONNECTED DIGRAPHS
}

\author{
Han Hyuk Cho and Hwa Kyung Kim
}

\begin{abstract}
Cho and Kim [4] and Kim [6] introduced the concept of the competition index of a digraph. Cho and Kim [4] and Akelbek and Kirkland [1] also studied the upper bound of competition indices of primitive digraphs. In this paper, we study the upper bound of competition indices of strongly connected digraphs. We also study the relation between competition index and ordinary index for a symmetric strongly connected digraph.
\end{abstract}

\section{Preliminaries and notations}

Let $D=(V, E)$ denote a digraph (directed graph) with vertex set $V=V(D)$, arc set $E=E(D)$. Loops are permitted but multiple arcs are not. A $x \rightarrow y$ walk in a digraph $D$ is a sequence of vertices $x, v_{1}, \ldots, v_{t}, y \in V(D)$ and a sequence of $\operatorname{arcs}\left(x, v_{1}\right),\left(v_{1}, v_{2}\right), \ldots,\left(v_{t}, y\right) \in E(D)$, where the vertices and arcs are not necessarily distinct. A closed walk is a $x \rightarrow y$ walk where $x=y$. A cycle is a closed $x \rightarrow y$ walk in which all vertices except $x$ and $y$ are distinct.

The length of a walk $W$ is the number of arcs in $W$. The notation $x \stackrel{k}{\rightarrow} y$ is used to indicate that there is a $x \rightarrow y$ walk of length $k$. The distance from vertex $x$ to vertex $y$ in $D$, denoted by $d(x, y)$, is the length of a shortest walk from $x$ to $y$. An $l$-cycle is a cycle of length $l$, and is denoted by $C_{l}$. If the digraph $D$ has at least one cycle, the length of a shortest cycle in $D$ is called the girth of $D$, and is denoted by $s(D)$.

A digraph $D$ is called strongly connected if for each pair of vertices $x$ and $y$ in $V(D)$ there is a walk from $x$ to $y$. For a strongly connected digraph $D$, the index of imprimitivity of $D$ is the greatest common divisor of the lengths of the cycles in $D$, and is denoted by $k(D)$. If $D$ is a trivial digraph of order $1, k(D)$ is undefined. For a strongly connected digraph $D, D$ is primitive if $k(D)=1$. If $D$ is primitive, there exists some positive integer $l$ such that there is a walk

Received October 23, 2009; Revised February 16, 2010.

2010 Mathematics Subject Classification. 05C20, 05C50.

Key words and phrases. competition graph, $m$-step competition graph, competition index, competition period, scrambling index, symmetric digraph.

The first author is supported by the Korean Research Foundation Grant (KRF-2005-015C00010). 
of length exactly $l$ from each vertex $x$ to each vertex $y$. The smallest such $l$ is called the exponent of $D$, denoted by $\exp (D)$. Exponents have been studied by many researchers $[2,7,8,9,10]$.

Cho et al. [5] introduced the $m$-step competition graph, a generalization of the competition graph. Let $D$ be a digraph (with or without loops) with the vertex set $\left\{v_{1}, v_{2}, \ldots, v_{n}\right\}$. Given a positive integer $m$, we say that a vertex $v_{k}$ of $D$ is an m-step common prey of $v_{i}$ and $v_{j}$ if there are two directed walks of length $m$, one from $v_{i}$ to $v_{k}$ and the other from $v_{j}$ to $v_{k}$. Then, the $m$-step competition graph of $D$, denoted by $C^{m}(D)$, has the same vertex set as $D$, and there is an edge between vertices $v_{i}$ and $v_{j}\left(v_{i} \neq v_{j}\right)$ if and only if $v_{i}$ and $v_{j}$ have an $m$-step common prey in $D$. The $m$-step digraph of $D$, denoted by $D^{m}$, has the same vertex set as $D$ and an $\operatorname{arc}\left(v_{i}, v_{j}\right)$ if and only if there is a directed walk of length $m$ from $v_{i}$ to $v_{j}$. Then we have $C^{m}(D)=C\left(D^{m}\right)$ for each positive integer $m$, see [5].

Consider the sequence $D, D^{2}, D^{3}, \ldots, D^{m}, \ldots$ Then there exists a smallest positive integer $q$ such that $D^{q}=D^{q+r}$ for some positive integer $r$. Such an integer $q$ is called the index of $D$ and is denoted by index $(D)$. There also exists a smallest positive integer $p$ such that $D^{q}=D^{q+p}$, and such an integer is called the period of $D$ and is denoted by period $(D)$. Now, consider the competition graph sequence $C(D), C\left(D^{2}\right), C\left(D^{3}\right), \ldots, C\left(D^{m}\right), \ldots$ There exists a smallest positive integer $q$ such that $C\left(D^{q+i}\right)=C\left(D^{q+r+i}\right)$ for some positive integer $r$ and every nonnegative integer $i$. Such an integer $q$ is called the competition index of $D$ and is denoted by $\operatorname{cindex}(D)$. Let $q=\operatorname{cindex}(D)$. Then there exists a smallest positive integer $p$ such that $C\left(D^{q+i}\right)=C\left(D^{q+p+i}\right)$ for every nonnegative integer $i$. Such an integer $p$ is called the competition period of $D$ and is denoted by cperiod $(D)$. From the definition of index and competition index, we get the following.

Proposition 1.1 (Cho and Kim [4]). For a digraph D, we have

$$
\text { cindex }(D) \leq \operatorname{index}(D) \text {. }
$$

Note that for a primitive digraph $D, \exp (D)=\operatorname{index}(D)$ and cindex $(D)$ is the smallest integer $q$ such that $C\left(D^{k}\right)$ is a complete graph for any $k \geq q$. It is well known that the index of imprimitivity of $D$ is equal to $\operatorname{period}(D)$ for a strongly connected digraph $D$ (For details on exponent (index) and period, refer to $[3,5,8,9,10])$. However, the competition period of a strongly connected digraph is always one, as shown below;

Proposition 1.2 (Cho and Kim [4]). For a strongly connected digraph D, we have

$$
\operatorname{cperiod}(D)=1 \text {. }
$$

Proof. Note that each vertex of $D$ has an outgoing arc. Thus, every edge in $C\left(D^{m}\right)$ is an edge in $C\left(D^{m+i}\right)$ for every positive integer $i$, since any two vertices having an $m$-step common prey also have an $(m+i)$-step common prey. Therefore, we have $\operatorname{cperiod}(D)=1$. 


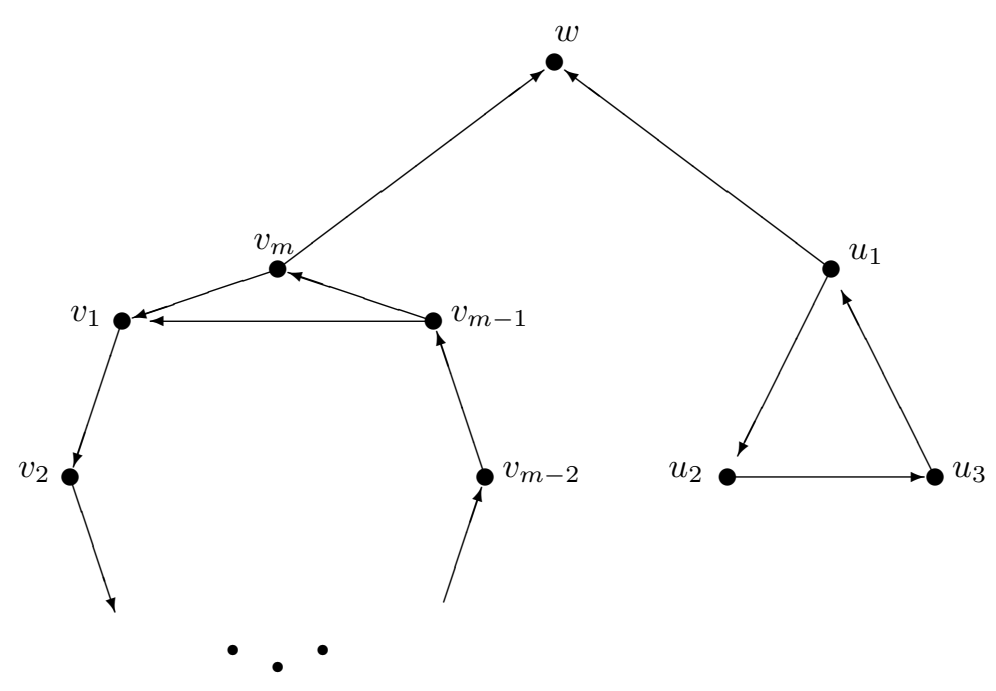

Figure 1. $U_{n}$ where $n=m+4$

Note that the competition period of a digraph may not be 1 when it is not strongly connected. For example, consider the above digraph $U_{n}$. Notice that there exist walks $u_{1} \stackrel{3 k-2}{\longrightarrow} w, u_{2} \stackrel{3 k}{\longrightarrow} w$ and $u_{3} \stackrel{3 k-1}{\longrightarrow} w$ for each positive integer $k$. Therefore we have the competition period of $U_{n}$ is not 1 , but 3 .

In [4], the upper bound of competition indices of primitive digraphs were studied as follows.

Lemma 1.3 (Cho and Kim [4]). Let $D$ be a primitive digraph $D$ of order $n(\geq 3)$ with girth $s$.

(1) If $n$ is odd, then cindex $(D) \leq n+\left(\frac{n-3}{2}\right) s$.

(2) If $n$ is even, then $\operatorname{cindex}(D) \leq n-1+\left(\frac{n-2}{2}\right) s$.

Akelbek and Kirkland [1] have introduced the scrambling index of a primitive digraph. Scrambling index is the smallest positive integer $k$ such that for every pair of vertices $u$ and $v$, there exists a vertex $w$ such that $u \stackrel{k}{\rightarrow} w$ and $v \stackrel{k}{\rightarrow} w$ in $D$. Akelbek and Kirkland's definition of scrambling index is the same as our definition of the competition index in the case of primitive digraphs. In [1], they presented the following result regarding the scrambling index.

Lemma 1.4 (Akelbek and Kirkland [1]). Let D be a primitive digraph with $n$ vertices and girth $s$.

(1) If $s$ is odd, then cindex $(D) \leq n-s+\left(\frac{s-1}{2}\right) n$.

(2) If $s$ is even, then cindex $(D) \leq n-s+\left(\frac{n-1}{2}\right) s$. 
Lemma 1.4 gives a better upper bound for the competition indices of primitive digraphs than Lemma 1.3.

For each integer $n(n \geq 3)$, a Wielandt digraph of order $n$ is a digraph isomorphic to $W_{n}$, as shown in below. Then $W_{n}$ is a primitive digraph with girth $n-1$, and $\operatorname{cindex}\left(W_{n}\right)=\omega_{n}$ where $\omega_{n}=\left[\frac{(n-1)^{2}+1}{2}\right]$ for a positive integer $n,[1,4]$.

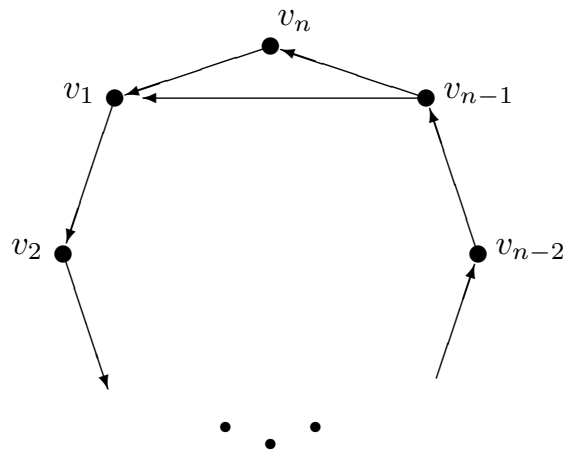

Figure 2. the Wielandt digraph $W_{n}$

Akelbek and Kirkland [1] and Cho and Kim [4] have considered the upper bound of the competition index of a primitive digraph as follows.

Theorem 1.5 (Akelbek and Kirkland [1], Cho and Kim [4]). Let D be a primitive digraph of order $n$. Then,

$$
\operatorname{cindex}(D) \leq\left\lceil\frac{(n-1)^{2}+1}{2}\right\rceil .
$$

Equality holds if and only if $D$ is a Wielandt digraph.

In Section 2, we consider the upper bound of the competition index of a strongly connected digraph. We also study the competition index of a symmetric digraph in Section 3.

\section{Competition indices of strongly connected digraphs}

We consider competition indices of strongly connected digraphs. Suppose $k=k(D) \geq 1$. Then, there exists a vertex partition $\left\{V_{1}, V_{2}, \ldots, V_{k}\right\}$ of the vertices $V$ of $D$ such that for each $1 \leq i \leq k$, every arc goes from $V_{i}$ to $V_{i+1}$ for some $i$, where $V_{k+1}=V_{1}$. Let $E_{i}$ be the subgraph of $D^{k}$ induced by $V_{i}$. Then, $E_{i}$ is primitive (refer to Theorem 3.4.5 in [2]). 
Consider the strongly connected digraph $T_{11}$ shown in Figure 3 . Note that $k\left(T_{11}\right)=2$. Let $V_{1}=\left\{v_{2}, v_{4}, v_{6}, v_{8}, v_{10}\right\}$ be a set of black vertices and $V_{2}=$ $\left\{v_{1}, v_{3}, v_{5}, v_{7}, v_{9}, v_{11}\right\}$ be a set of white vertices. Then, $\left\{V_{1}, V_{2}\right\}$ forms a vertex partition of $T_{11}$.

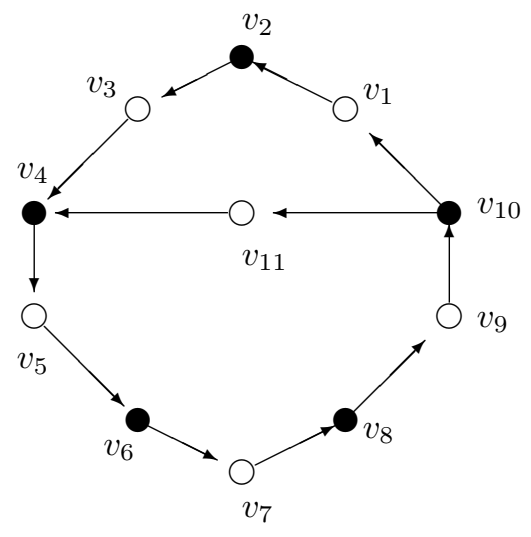

FiguRE 3. Digraph $T_{11}$ with index of imprimitivity 2

Now, consider the induced subgraph $E_{1}$ (respectively, $E_{2}$ ) of $V_{1}$ (respectively, $V_{2}$ ) in the 2-step digraph $T_{11}^{2}$ of $T_{11}$ as shown in Figure 4 . Then $T_{11}^{2}$ is a disjoint union of $E_{1}$ and $E_{2}$. Note that $E_{1}$ is a Wielandt digraph of order 5 . We also have $\operatorname{cindex}\left(E_{1}\right)=9$ and $\operatorname{cindex}\left(E_{2}\right)=10$. Furthermore, we have $\operatorname{cindex}\left(T_{11}\right)=19$.

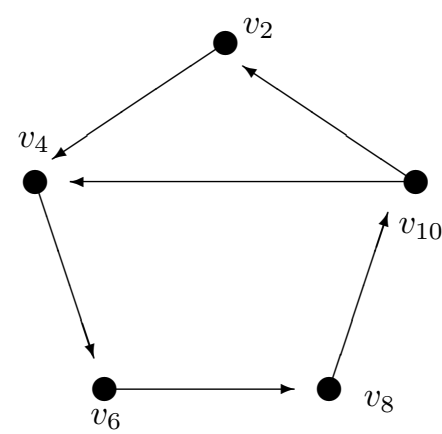

$E_{1}$

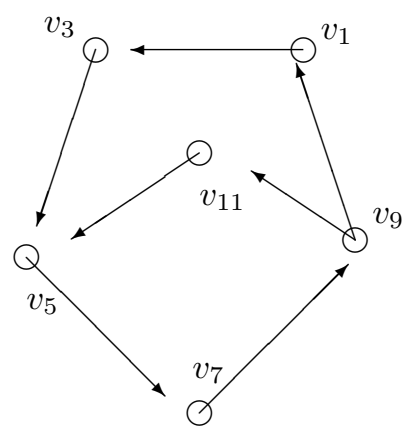

$E_{2}$

Figure 4. Its induced subgraphs $E_{1}, E_{2}$ 
Let $D$ be a strongly connected digraph. For a pair of vertices $u$ and $v$ having a $k$-step common prey, let $\operatorname{cindex}(D: u, v)$ denote the smallest positive integer $k$ such that $u$ and $v$ have a $k$-step common prey. If $u$ and $v$ do not have any $k$-step common prey, we let $\operatorname{cindex}(D: u, v)=1$. We also let $\operatorname{cindex}(D: u, u)=1$. Let $\operatorname{cindex}(D: u)$ denote $\max \{\operatorname{cindex}(D: u, v) \mid v \in V(D)\}$. Then we have cindex $(D)=\max \{\operatorname{cindex}(D: u) \mid u \in V(D)\}$ and $\operatorname{cindex}(D)=\max \{\operatorname{cindex}(D$ : $u, v) \mid u, v \in V(D)\}$.

Now, let $D$ be a primitive digraph. For a pair of vertices $u$ and $v$ in $V(D)$, let $\exp (D: u, v)$ denote the smallest integer $k$ such that there exists a directed walk of length $t$ from $u$ to $v$ in $D$ for all $t \geq k$, and let $\exp (D: u)=\max \{\exp (D$ : $u, v) \mid v \in V(D)\}$. Then, we have $\exp (D)=\max \{\exp (D: u, v) \mid u, v \in V(D)\}$.

Theorem 2.1. Suppose $k(D)=k$ for a strongly connected digraph $D$. Let $V_{1}$, $V_{2}, \ldots, V_{k}$ be $k$ nonempty sets, with $V_{k+1}=V_{1}$, where each arc of $D$ issues from $V_{i}$ and enters $V_{i+1}$ for some $i$ with $1 \leq i \leq k$. Let $E_{i}$ be the subgraph of $D^{k}$ induced by $V_{i}$, where $1 \leq i \leq k$, and $m=\min \left\{\operatorname{cindex}\left(E_{i}\right) \mid i=1,2, \ldots, k\right\}$ in $D^{k}$. Then, we have

$$
\operatorname{cindex}(D) \leq k(m+1)-1 .
$$

Proof. We show that $\operatorname{cindex}(D: u, v) \leq k(m+1)-1$ for any two vertices $u$ and $v$. If $u \in V_{i}, v \in V_{j}$, and $i \neq j$, then $u$ and $v$ do not have an $l$-step common prey for any positive integer $l$. Thus, $\operatorname{cindex}(D: u, v)=1$. Suppose $u, v \in V_{j}$ and $m=\operatorname{cindex}\left(E_{r}\right)$ for some $1 \leq j, r \leq k$. Let $u^{\prime}, v^{\prime}$ be vertices in $V_{r}$ such that there exist walks $u \stackrel{f}{\rightarrow} u^{\prime}$ and $v \stackrel{f}{\rightarrow} v^{\prime}$, where $0 \leq f \leq k-1$. Since $\operatorname{cindex}\left(D^{k}: u^{\prime}, v^{\prime}\right) \leq \operatorname{cindex}\left(E_{r}\right)$, we have

$$
\begin{aligned}
\operatorname{cindex}(D: u, v) & \leq f+\operatorname{cindex}\left(D: u^{\prime}, v^{\prime}\right) \\
& \leq k-1+k \cdot \operatorname{cindex}\left(D^{k}: u^{\prime}, v^{\prime}\right) \\
& \leq k-1+k \cdot \operatorname{cindex}\left(E_{r}\right) .
\end{aligned}
$$

We have $\operatorname{cindex}(D: u, v) \leq k(m+1)-1$. Thus, we have $\operatorname{cindex}(D)=$ $\max \{\operatorname{cindex}(D: u, v) \mid u, v \in V(D)\} \leq k(m+1)-1$. This establishes the result.

Note that the inequality in Theorem 2.1 is sharp. For the digraph $D=T_{11}$ shown in Figure 3, we have that $k=2, m=9=\operatorname{cindex}\left(E_{1}\right)$, and $\operatorname{cindex}\left(T_{11}\right.$ : $\left.v_{1}, v_{5}\right)=19$. Thus, we have cindex $\left(T_{11}\right)=k(m+1)-1$.

Lemma 2.2. Let $D$ be a strongly connected digraph of order $n(\geq 3)$. If $k(D)>\frac{n}{2}$, then we have

$$
\operatorname{cindex}(D) \leq\left\lfloor\frac{n-1}{2}\right\rfloor .
$$

Proof. Let the girth of $D$ be $s$. If $k(D)>\frac{n}{2}$, then every cycle in $D$ has the same length $s(=k(D))$. Let $V_{1}, V_{2}, \ldots, V_{s}$ be $s$ nonempty sets, with $V_{s+1}=V_{1}$, where each arc of $D$ issues from $V_{i}$ and enters $V_{i+1}$ for some $i$ with $1 \leq i \leq s$. 
Consider two vertices $u \in V_{i}$ and $y \in V_{j}$. If $i \neq j$, or $i=j$ and $u=v$, then $\operatorname{cindex}(D: u, v)=1$. If $u$ and $v$ do not have $l$-step common prey for any positive number $l$, then $\operatorname{cindex}(D: u, v)=1$. Without loss of generality, we may assume that $\operatorname{cindex}(D: u, v)=m>1$ and $u, v \in V_{s}$. Then we have walks such that $u \rightarrow u_{1} \rightarrow \cdots \rightarrow u_{m}=w$ and $v \rightarrow v_{1} \rightarrow \cdots \rightarrow v_{m}=w$, where $u_{i} \neq v_{i}$ and $u_{i}, v_{i} \in V_{i}(i=1, \ldots, m-1)$. Therefore we have $2 m+1 \leq n$ and $\operatorname{cindex}(D: u, v)=m \leq\left\lfloor\frac{n-1}{2}\right\rfloor$. This establishes the result.

From Theorem 2.1 and Lemma 2.2, we have the following theorem.

Theorem 2.3. Suppose $D$ is a strongly connected digraph of order $n(\geq 3)$. Then, we have

$$
\operatorname{cindex}(D) \leq \omega_{n}=\left\lceil\frac{(n-1)^{2}+1}{2}\right\rceil .
$$

This equality holds if and only if $D$ is isomorphic to $W_{n}$.

Proof. Let $k(D)=k$. If $k=1$, the above equality holds by Theorem 1.5. If $n=3$ and $k \geq 2$, we can check that cindex $(D) \leq \omega_{n}$. If $k(D)>\frac{n}{2}$, this equality holds by Lemma 2.2. Assume that $2 \leq k \leq \frac{n}{2}$ and $n \geq 4$. Let $V_{1}$, $V_{2}, \ldots, V_{k}$ be $k$ nonempty sets, with $V_{k+1}=V_{1}$, where each $\operatorname{arc}$ of $D$ issues from $V_{i}$ and enters $V_{i+1}$ for some $i$ with $1 \leq i \leq k$. Let $E_{i}$ be the subgraph of $D^{k}$ induced by $V_{i}$, where $1 \leq i \leq k$. Then, there exists a positive integer $r$ such that the order of $E_{r}$ is less than or equal to $\left\lfloor\frac{n}{k}\right\rfloor$. From Theorem 1.5, we have cindex $\left(E_{i}\right) \leq \omega_{\left|V_{i}\right|}$ for each $1 \leq i \leq k$ since each $E_{i}$ is a primitive digraph. Therefore we have $\operatorname{cindex}\left(E_{r}\right) \leq \omega_{\left\lfloor\frac{n}{k}\right\rfloor}$. By Theorem 2.1, we have

$$
\begin{aligned}
\operatorname{cindex}(D) & \leq k-1+k \cdot \operatorname{cindex}\left(E_{r}\right) \\
& \leq k-1+k \cdot \omega_{\left\lfloor\frac{n}{k}\right\rfloor} \\
& \leq k-1+k \cdot \frac{\left(\frac{n}{k}\right)^{2}-2\left(\frac{n}{k}\right)+3}{2} \\
& =\frac{n^{2}}{2 k}+\frac{5}{2} k-n-1 .
\end{aligned}
$$

Let $g(k)=\frac{n^{2}}{2 k}+\frac{5}{2} k-n-1\left(2 \leq k \leq \frac{n}{2}\right)$. Then $g(k)$ attains the maximum value when $k=2$. $g(2)=\frac{n^{2}-4 n+16}{4}<\omega_{n}$ since $n \geq 4$. Therefore, we have $\operatorname{cindex}(D)<\omega_{n}$ for a nonprimitive strongly connected digraph $D$. This establishes the result.

Next we study the competition indices of symmetric strongly connected digraphs.

\section{Competition indices of symmetric strongly connected digraphs}

In this section, we assume that $D$ is a symmetric strongly connected digraph of order $n(\geq 2)$. Observe that in symmetric graphs, strongly connected is the same as connected. Since there is a 2 -cycle in $D$, we have $k(D) \leq 2$. For 
a connected symmetric primitive digraph $D, k(D)=2$ if $D$ is bipartite, and otherwise $k(D)=1$.

Lemma 3.1 (Shao [8]). Let $u$ and $v$ be vertices of a symmetric primitive digraph $D$ such that there exist directed walks of lengths $k_{1}$ and $k_{2}$, respectively, with different parity between $u$ and $v$. Then, we have

$$
\exp (D: u, v) \leq \max \left\{k_{1}, k_{2}\right\}-1 \text {. }
$$

Proposition 3.2 (Shao [8]). Let $E_{n}=\{\exp (D): D$ is a symmetric primitive digraph of order $n \geq 4\}$. Then we have

$$
E_{n}=\{1,2, \ldots, 2 n-2\} \backslash S,
$$

where $S$ is the set of all odd numbers in $\{n, n+1, \ldots, 2 n-3\}$.

Lemma 3.3. Let $D$ be a symmetric primitive digraph of order $n$. Then, for each vertex $u$, we have

$$
\operatorname{cindex}(D: u)=\left\lceil\frac{\exp (D: u)}{2}\right\rceil .
$$

Proof. Consider two vertices $u$ and $v$ in $V(D)$.

First, we have two directed walks $u \stackrel{\exp (D: u, v)}{\longrightarrow} v$ and $u \stackrel{\exp (D: u, v)+1}{\longrightarrow} v$. Since $D$ is symmetric, $u$ and $v$ have a $\left[\frac{\exp (D: u, v)}{2}\right]$-step common prey. Therefore, we have

$$
\operatorname{cindex}(D: u, v) \leq\left\lceil\frac{\exp (D: u, v)}{2}\right\rceil \text {. }
$$

Suppose $u=v$. Then there exists a vertex $w(\neq u)$ such that $u \stackrel{1}{\rightarrow} w$, and there exists a directed walk $u \stackrel{2 \cdot \operatorname{cindex}(D: u, w)}{\longrightarrow} w$. From Lemma 3.1, we have $\exp (D: u, w) \leq 2 \cdot \operatorname{cindex}(D: u, w)-1$. Therefore, we have

$$
\exp (D: u, v) \leq \exp (D: u, w)+1 \leq 2 \cdot \operatorname{cindex}(D: u, w) \leq 2 \cdot \operatorname{cindex}(D: u) .
$$

Suppose $u \neq v$. If $d(u, v)$ is odd, then we have two walks $u \stackrel{d(u, v)}{\longrightarrow} v$ and $u \stackrel{2 \cdot \operatorname{cindex}(D: u, v)}{\longrightarrow} v$. From Lemma 3.1, we have $\exp (D: u, v) \leq 2 \cdot \operatorname{cindex}(D: u)-1$ since $2 \cdot \operatorname{cindex}(D: u)>d(u, v)$. If $d(u, v)$ is even, then we have a vertex $w$ such that $u \stackrel{d(u, v)-1}{\longrightarrow} w(\neq u) \rightarrow v$. We also have $u \stackrel{2 \cdot \operatorname{cindex}(D: u, w)}{\longrightarrow} w$. Since $d(u, v)-1$ is odd, we have $\exp (D: u, w) \leq 2 \cdot \operatorname{cindex}(D: u, w)-1$. Therefore, $\exp (D: u, v) \leq \exp (D: u, w)+1 \leq 2 \cdot \operatorname{cindex}(D: u, w) \leq 2 \cdot \operatorname{cindex}(D: u)$. $\exp (D: u, v) \leq 2 \cdot \operatorname{cindex}(D: u)$. Therefore, we always have

$$
\frac{\exp (D: u)}{2} \leq \operatorname{cindex}(D: u)
$$

From the above results, we have $\frac{\exp (D: u)}{2} \leq \operatorname{cindex}(D: u) \leq\left\lceil\frac{\exp (D: u)}{2}\right\rceil$. For each vertex $u$ in $D$, we have

$$
\operatorname{cindex}(D: u)=\left\lceil\frac{\exp (D: u)}{2}\right\rceil .
$$


This establishes the result.

Theorem 3.4. If $D$ is a symmetric strongly connected digraph, then we have

$$
\operatorname{cindex}(D)=\left\lceil\frac{\operatorname{index}(D)}{2}\right\rceil \text {. }
$$

Proof. Let the order of $D$ be $n$. If $n=1$, this equality holds. Suppose $n \geq 2$. Then, we have $k(D) \leq 2$.

Case 1. $k(D)=1$

Note that $\operatorname{index}(D)=\max \{\exp (D: u)\}$ and $\operatorname{cindex}(D)=\max \{\operatorname{cindex}(D: u)\}$. From Lemma 3.3, the result is established.

Case 2. $k(D)=2$

There are bipartition $V_{1}$ and $V_{2}$ of the vertex set $V(D)$. Consider two vertices $u \in V_{i}$ and $v \in V_{j}$.

If $i \neq j$, we have $\operatorname{cindex}(D: u, v)=1$. If $i=j$ and index $(D)$ is even, there is a directed walk such that $u \stackrel{\operatorname{index}(D)}{\longrightarrow} v$. Then, we have $\operatorname{cindex}(D: u, v) \leq$ $\frac{\operatorname{index}(D)}{2}$. If $i=j$ and $\operatorname{index}(D)$ is odd, there is a directed walk such that $u \stackrel{\operatorname{index}(D)+1}{\longrightarrow} v$. In all cases, we have $\operatorname{cindex}(D: u, v) \leq \frac{\operatorname{index}(D)+1}{2}$. Therefore, we have $\operatorname{cindex}(D) \leq\left\lceil\frac{\operatorname{index}(D)}{2}\right\rceil$.

If $i=j$, there exists $u \stackrel{2 \cdot \operatorname{cindex}(D)}{\longrightarrow} v$. If $i \neq j$, there is no $u \stackrel{2 \cdot \operatorname{cindex}(D)}{\longrightarrow} v$ and there exist a vertex $u^{\prime} \in V_{j}$ and a directed walk such that $u \stackrel{1}{\longrightarrow} u^{\prime} \stackrel{2 \cdot \operatorname{cindex}(D)}{\longrightarrow} v$. Then, we have $D^{2 \cdot \operatorname{cindex}(D)}=D^{2 \cdot \operatorname{cindex}(D)+2}$. Therefore, we have index $(D) \leq$ $2 \cdot \operatorname{cindex}(D)$.

This establishes the result.

Corollary 3.5. If $D$ is a symmetric primitive digraph of order $n \geq 4$, then cindex $(D) \leq n-1$, and equality holds if and only if $D$ is a path with a loop on an end vertex.

Proof. From Proposition 3.2 and Theorem 3.4, we have cindex $(D)=\left\lceil\frac{\operatorname{index}(D)}{2}\right\rceil$ $\leq n-1$. Since there are no symmetric primitive digraphs whose index is $2 n-3$ from Proposition 3.2, this corollary is proved.

\section{Closing remark}

In this paper, we have introduced the concept of the competition index of a digraph using the concept of the $m$-step competition graph introduced in [5]. For digraphs whose competition periods are one, there are some similarities between the property of the competition indices and that of ordinary indices or exponents. However, for digraphs whose competition periods are not one, there are significant differences between their competition indices and ordinary indices. In the case of the ordinary index, it is well known that index $(D) \leq$ $(n-2)^{2}+2$ for a reducible digraph $D$ of order $n,[7,9]$. This implies that the 
index of a Wielandt digraph is the maximum possible index among indices of digraphs of order $n$. However, in the case of a competition index, the similarity between the properties does not hold. For example, consider the competition index of $U_{n}$ shown in Figure 1. Then, we have cindex $\left(U_{n}\right)=m^{2}-2 m+3$ and $\operatorname{cperiod}\left(U_{n}\right)=3$. Thus cindex $\left(U_{n}\right)>\omega_{n}$ when $n \geq 15$. That is, there is a reducible digraph of order $n$ whose competition index is greater than that of any strongly connected digraph of order $n$. As shown in the example $U_{n}$, the competition indices of reducible digraphs are very interesting, and there is much work to be done.

Acknowledgement. We would like to thank an anonymous referee for his or her useful suggestions.

\section{References}

[1] M. Akelbek and S. Kirkland, Coefficients of ergodicity and the scrambling index, Linear Algebra Appl. 430 (2009), no. 4, 1111-1130.

[2] R. A. Brualdi and H. J. Ryser, Combinatorial Matrix Theory, Cambridge University Press, 1991.

[3] H. H. Cho, Indices of irreducible Boolean matrix, J. Korean Math. Soc. 30 (1993), no. $2,267-274$.

[4] H. H. Cho and H. K. Kim, Competition indices of digraphs, Proceedings of workshop in combinatorics (2004), 99-107.

[5] H. H. Cho, S.-R. Kim, and Y. Nam, The m-step competition graph of a digraph, Discrete Appl. Math. 105 (2000), no. 1-3, 115-127.

[6] H. K. Kim, Competition indices of tournaments, Bull. Korean Math. Soc. 45 (2008), no. 2, 385-396.

[7] B. Liu and H.-J. Lai, Matrices in Combinatorics and Graph Theory, Kluwer Academic Publishers, 2000.

[8] J. Shao, The exponent set of symmetric primitive matrices, Scientia Sinica Ser. A 30 (1987), no. 4, 348-358.

[9] J. Shao and Q. Li, The indices of convergence reducible Boolean matrices, Acta Math. Sinica 33 (1990), 13-28.

[10] J. Shen, Proof of a conjecture about the exponent of primitive matrics, Linear Algebra and Its Appl. 216 (1995), 185-203.

HAN HYUK CHO

Department of Mathematics Education

Seoul National University

SEOUl 151-748, Korea

E-mail address: hancho@snu.ac.kr

HWA KYUNG Kim

Department of Mathematics Education

SANGMYUNG UNIVERSITY

SeOul 110-743, KoreA

E-mail address: indices@smu.ac.kr 